\title{
El concepto del límite y su aplicación en algunas operaciones financieras
}

\author{
The concept of the limit and its application on some financial transations
}

\author{
Omar Enrique Valencia Angulo ${ }^{1}$, Edgar Alirio Valencia Angulo ${ }^{2}$ \\ ${ }^{1}$ Administrador de empresas de la Universidad del Valle; especialista en gerencia financiera de la \\ Universidad Central, docente capacitador y asesor en temas administrativos y financieros, Cali, Colombia. \\ ${ }^{2}$ Departamento de Matemáticas, Universidad Tecnológica de Pereira, Pereira, Colombia \\ Correo-e: omeva2003@yahoo.com. \\ Correo-e: evalenciadutp.edu.co
}

\begin{abstract}
Resumen- El artículo explica cómo se aplica el concepto del límite matemático en operaciones financieras acudiendo a recursos teórico/prácticos, como son, por un lado los principios que lo definen y de otra parte, una oferta de ejemplos resueltos para ilustrar; como resultado, se puede demostrar no solo que a medida que la variable independiente varía, la variable dependiente se acerca a un valor acotado o límite; donde la variable independiente es el número de periodos (sinónimo de tiempo), mientras que la variable dependiente es el valor objetivo en cuestión (valor presente o valor futuro); también se establece que en el caso de los inversionistas, obtienen mayores beneficios de contar con una mayor composición (número de periodos).
\end{abstract}

Palabras clave-Límite, interés compuesto, función exponencial, logaritmo, variable independiente, variable dependiente, capitalización, número de Euler, interés periódico, interés nominal, Tasa Efectiva Anual, interés continuo.

Abstract_ The article explains how it applies the concept of the mathematical limit on financial operations; using resources theoretical/practical, as they are, on the one hand the principles that define it and on the other hand, a range of examples resolved to illustrate; as a result, it can be shown not only to the independent variable varies, the dependent variable is approaching a bounded value (limit); where is the independent variable the number of periods (synonym of time), while the dependent variable is the target value in question (present or future value); He is also established that in the case of investors, obtain greater benefits from having a major composition (number of periods).

Key Word Limit, compound interest, exponential function, logarithm, independent variable, dependent variable, capitalization, number of Euler, periodic interest, coupon rate, effective annual rate, continued interest.

\section{INTRODUCCIÓN}

En el presente artículo se busca explicar el concepto del límite, luego se establece que las operaciones con aplicación del concepto del límite, están ligadas al número real e, al que Sobel y Lerner [1] lo definen como "el número real que permite que la tangente a la curva definida por $\mathrm{y}=\boldsymbol{e}^{\boldsymbol{x}}\{\mathbf{1}\}$, en el punto $(0,1)$ ", como se refleja en el gráfico $\# 1$; de otra forma en el libro Cálculo de una variable de Thomas, Jr. G. B. [2] dice que es el único valor que satisface la siguiente ecuación: $\ln (\mathrm{e})=1$. $\{2\}$.

\section{Gráfico \#1: FUNCION EXPONENCIAL CON BASE e}

$$
y=e^{x}
$$

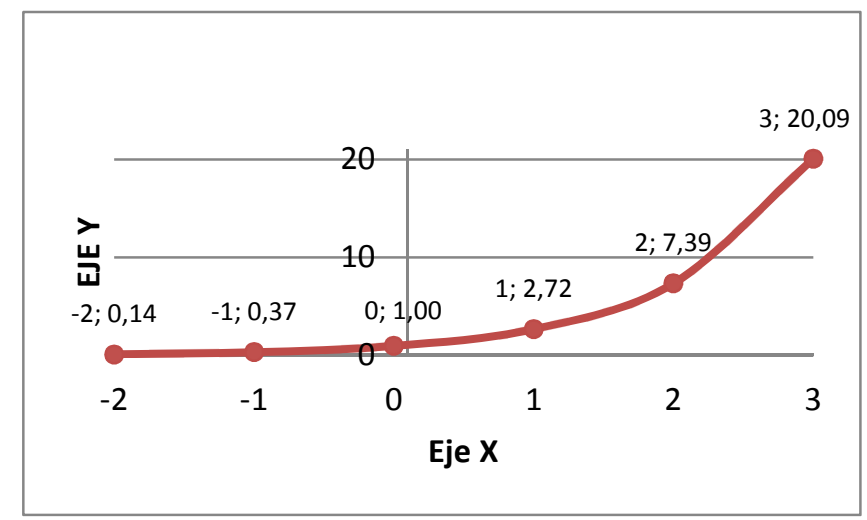

Fuente: Sobel y Lerner [1].

En la financiación de bienes y servicios un componente que marca este tipo de negociaciones, es la frecuencia con la cual se causan los intereses o los pagos respectivos, como es la capitalización; cuando la frecuencia de la capitalización es intensa, el uso de las herramientas financieras usuales 
(fórmulas), se desvirtúa un poco, por lo cual es más eficiente, más sencillo y preciso, el uso de la función exponencial con base e.

La constante matemática e - de valor aproximado a 2.71828182846-, es un número de mucha utilidad en ciertas funciones, especialmente, la potencia, exponencial y su inversa, la logarítmica; también se conoce como número Euler, en honor al matemático suizo Leonard Euler; y su valor puede obtenerse a partir de la siguiente fórmula: $\mathrm{e}=\left(1+\frac{1}{n}\right)^{n}$ $\{3\}$ en la medida que $n$ sea un número muy grade grande, como se demuestra en la siguiente tabla:

Tabla 1: Acotamiento del valor en la fórmula Cuando $\mathrm{n}$ crece de forma exponencial.

$\frac{n}{\left(1+\frac{1}{n}\right)^{n}}\left|\frac{1}{2}\right|\left|\frac{10}{2.59}\right|\left|\frac{100}{2.705}\right|\left|\frac{1,000}{2.7169}\right|\left|\frac{10,000}{2.71815}\right|\left|\frac{100,000}{2.718268}\right|\left|\frac{1^{\prime} 000,000}{2.7182804}\right|$

Fuente: Hoffmann, L. D. [3]

Y que se puede visualizar en el siguiente gráfico

Gráfico 2: Acotamiento del valor en la fórmula

Cuando $\mathrm{n}$ crece de forma exponencial.

$$
\mathrm{Y}=\left(\mathbf{1}+\frac{1}{n}\right)^{n}
$$

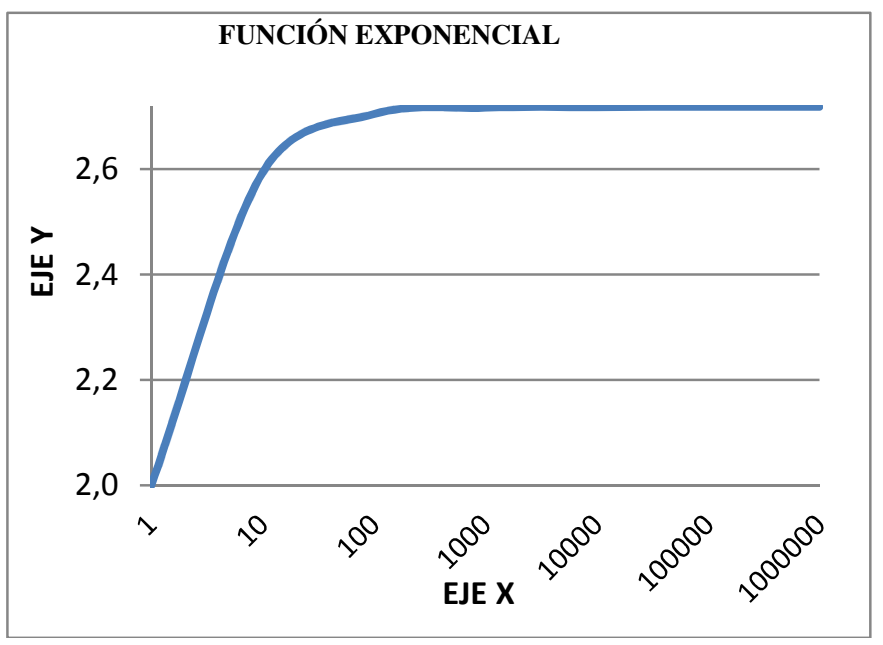

Los datos encontrados permiten establecer una generalización, en la cual e $=\lim _{n \rightarrow \infty}\left(1+\frac{1}{n}\right)^{n}\{4\}$ tal como lo ilustra Bittinger, M. L. [4].

Vale la pena recordar que la función exponencial, explica operaciones del tipo $f(x)=\boldsymbol{a}^{\boldsymbol{x}}$, donde $\boldsymbol{a}$ es una constante positiva (un valor asignado), y $x$ es una variable (independiente) y el resultado equivale a la variable dependiente.
En la argumentación del presente documento, además de las referencias enunciadas, se ha acudido a los textos de Domínguez, Carlos [5], Corredores y asociados [6], Vélez, P. I. [7], Aching, G. C. [8], Haeussler E. F. Jr y Richard, S. P. [9], Aguirre, V. H. M. [10] y Varela V. R. [11].

\section{APLICACIONES DE LA FUNCION EXPONENCIAL DE BASE E EN OPERACIONES FINANCIERAS}

La financiación de capitales involucra una serie de conceptos sobre los cuales es menester tener claridad, entre los cuales se hallan.

Concepto 1: En cuanto al interés (valor corriente, es decir expresado en alguna moneda transable), este es la materialización por el uso de recursos cuantificables (en dinero) durante cierto espacio de tiempo y resulta de aplicar el valor determinado como porcentaje de interés (tasa de interés), al capital en cuestión; establecido lo anterior, ese porcentaje de interés puede expresarse como interés periódico o como interés nominal y tiene su equivalencia respectiva.

El interés periódico, es propiamente simbolizado con la letra $\mathbf{i}$, mientras que el interés nominal, es más frecuente encontrarlo como in y su equivalencia se demuestra en la siguiente igualdad: $\mathrm{i}=\frac{\boldsymbol{i n}}{\boldsymbol{m}}\{5\}$; los componentes de esta fórmula se describen y explican muy bien, en detalle en el libro de Domínguez, Carlos [5]. En la que se entiende que el interés periódico es igual al interés nominal dividido entre el número de periodos en un año, de acuerdo a la composición.

Concepto 2: En las transacciones financieras, cuando se presenta el interés nominal y con él se hace necesario obtener el interés periódico, dividiendo el primero entre el número de periodos, en un año de acuerdo a la composición, a menudo se utiliza $\mathbf{m}$, que se diferencia de $\mathbf{n}$, en que esta última, representa los periodos totales dentro de la operación crediticia

Concepto 3: Las secuencias iterativas, son procesos que describen actividades repetitivas en general (un proceso genérico per se) o actividades específicas de repetición, pero con cambio o variación en por lo menos una variable.

Concepto 4: Dentro del interés compuesto y en relación a la capitalización, existen el Interés Compuesto Discreto, aquel en el cual los periodos recurrentes cuando se capitaliza la inversión, son conocidos y claramente expresados (anual, semestral, tetramestral, trimestral, bimestral, mensual, etc. Pero además existe el Interés Continuo, que define aquel en que los periodos de capitalización, son mucho más frecuentes, siendo a veces difícil de cuantificar o establecer, lo que implica una capitalización más intensa (cada día, cada minuto, cada segundo, etc.) 
Formulación: Para establecer el TAE en una operación financiera, existe una igualdad como la siguiente: T.A.E. = $\left[(\mathbf{1}+\boldsymbol{i})^{\boldsymbol{m}}\right]-\mathbf{1} .\{\mathbf{6}\}$. Tomada del texto de Corredores y asociados S. A. [6].

Mientras que para interés continuo se utiliza la siguiente ecuación, como se ilustra en el texto de Vélez, P. I. [7]: T.A.E. $=\left(\mathbf{e}^{i n}\right)-\mathbf{1} .\{7\}$.

Ahora, resulta que en la ecuación $\{6\}$, la variable $\mathbf{i}$ se puede obtener de otra forma, de acuerdo a la ecuación $\{5\}$, que es muy similar a la manera como la muestra Aching, G. C. [8]:

$$
\text { T.A.E. }=\left[\left(1+\frac{i n}{m}\right)^{m}\right]-1 .\{8\} \text {. }
$$

Ahora se determinará la T.A.E. con in $=18 \%$, para composición anual, semestral, tetramestral, trimestral, bimestral, mensual, semanal, diaria, por horas, aplicando la igualdad $\{8\}$

T.A.E. de composición anual

$$
\left[\left(1+\frac{0.18}{1}\right)^{1}\right]-1=0.18
$$

T.A.E. de composición semestral:

$$
\left[\left(1+\frac{\mathbf{0 . 1 8}}{\mathbf{2}}\right)^{2}\right]-1=0.1881
$$

T.A.E. de composición tetramestral:

$$
\left[\left(1+\frac{0.18}{3}\right)^{3}\right]-1=0.191016
$$

T.A.E. de composición trimestral:

$$
\left[\left(1+\frac{0.18}{4}\right)^{4}\right]-1=0.1925186
$$

T.A.E. de composición bimestral:

$$
\left[\left(1+\frac{0.18}{6}\right)^{6}\right]-1=0.194052
$$

T.A.E. de composición mensual:

$$
\left[\left(1+\frac{0.18}{12}\right)^{12}\right]-1=0.195618
$$

T.A.E. de composición semanal:

$$
\left[\left(1+\frac{0.18}{52}\right)^{52}\right]-1=0.1968453
$$

T.A.E. de composición diaria:

$\left[\left(1+\frac{0.18}{365}\right)^{365}\right]-1=0.1971639$

T.A.E. de composición por horas:

$$
\left[\left(1+\frac{0.18}{8760}\right)^{8760}\right]-1=0.1972147
$$

Como se puede observar, al determinar una composición mayor, es decir una capitalización más intensa, la tasa de interés efectiva, tiende a moverse poco respecto de un valor, en este caso $19.72 \%$ T.A.E.

Ahora, se resolverá aplicando la fórmula \{7\}: T.A.E. = $\left(\mathbf{e}^{i n}\right)-\mathbf{1}$, para operaciones pactadas a interés continuo:

T.A.E. $=\left(\mathbf{e}^{\mathbf{0 . 1 8}}\right)-\mathbf{1}=0.1972174$.

Los resultados corroboran porque, cuando una operación financiera tiene una alta composición o una intensa capitalización, para determinar la T.A.E, es mejor utilizar la ecuación para interés continuo.

Ahora se pasa a develar porque las dos igualdades tienden a representar un valor similar cuanto $\mathrm{m}$ tiende a crecer sin límite, para ello se retoma la ecuación $\{8\}$ :

$$
\text { T.A.E. }=\left[\left(1+\frac{i n}{m}\right)^{m}\right]-1 .
$$

Se puede observar claramente que el interés para cada periodo en consideración es $\frac{\boldsymbol{i n}}{\boldsymbol{m}}$; y dado que $m$ representa los periodos de composición en un año y al ser in un valor inferior a uno, que se hace aún más pequeño al dividirse entre $\mathrm{m}$, es lo que exige adicionar el número uno, para poder multiplicar periodo a periodo, porque de no ser así, el producto cada vez sería más pequeño, y ello no es razonable (una operación financiera, donde el interés es menor a un mayor tiempo); luego, al encontrar el factor, se le resta el mismo número uno y queda la tasa encontrada. Ahora, se reitera que el valor encontrado es la T.A.E (Tasa Anual Efectiva), pero de ser necesario encontrar la tasa efectiva de varios años, el rendimiento efectivo está representado así:

$$
\text { T.E. }(\text { Tasa Efectiva })=\left[\left(1+\frac{\boldsymbol{i n}}{m}\right)^{m t}\right]-1 \text {. }
$$

Donde $t$ significa el número de años.

A continuación, para simplificar el cálculo y la expresión, se puede apelar a que $n=\frac{\boldsymbol{m}}{\boldsymbol{i n}}\{10\}$, lo que llevaría a que $\boldsymbol{m}=n * i n$ $\{11\}$, lo que permitiría establecer en la ecuación $\{9\}$ : 
T.E. $=\left[\left(1+\frac{i n}{m}\right)^{m t}\right]-1 \rightarrow$ Se simplifica la fracción $\frac{i n}{m}$ así: $\frac{i n}{i n}$ $=1 \mathrm{y} \frac{m}{\boldsymbol{i n}}=n$; al tiempo que $m=n *$ in para transformar la fórmula:

$\left[\left(\mathbf{1}+\frac{\mathbf{1}}{\boldsymbol{n}}\right)^{\boldsymbol{n} * \boldsymbol{i n} * \boldsymbol{t}}\right]-1 . \quad\{10\} \quad$ Esta ecuación se podría expresar de la siguiente forma:

$\left|\left[\left(\mathbf{1}+\frac{\mathbf{1}}{n}\right)^{n}\right]^{i n * t}\right|-\mathbf{1}\{\mathbf{1 1}\} \quad \mathrm{Y}$ ya se ha demostrado que $\left(\mathbf{1}+\frac{\mathbf{1}}{n}\right)^{n}$ tiende a e, cuando $n$ crece sin límite, por lo que se concluye que: $\left[\left(\mathbf{1}+\frac{\boldsymbol{i n}}{\boldsymbol{m}}\right)^{\boldsymbol{m} t}\right]-1$ tiende a $\left(\mathbf{e}^{\boldsymbol{m} t}\right)-\mathbf{1}$ cuando $m$ crece sin límite

Este análisis y explicación se puede observar un poco más detallado en el libro de Hoffmann L. D. [3].

Adicionalmente el texto de. Haeussler E. F. Jr. y Richard, S. P. [9], describe que se puede analizar, la ecuación desde otra perspectiva para llegar al mismo valor, y esto es que al $n$ crecer sin límite, se puede generalizar que $n \rightarrow \infty\{12\}$, por lo cual, la expresión entre paréntesis, en la formula $\{11\}$, tiene la forma $\mathrm{e}=\lim _{x \rightarrow \emptyset}(1+x)^{(1 / x)}\{13\}$, dado que el factor $\frac{1}{n}$ se puede simplificar y obtener $\frac{\mathbf{1}}{\boldsymbol{n}}=x\{14\}$, por lo mismo $n=\frac{\mathbf{1}}{\boldsymbol{x}}$ $\{15\}$

Ejemplo 1. El director de mercadeo del banco del Comercio está diseñando una estrategia comercial para captar recursos de ahorradores e inversionistas a una tasa nominal del $15 \%$ y desea visualizar en compañía del gerente, el impacto de la capitalización al contemplar diferentes alternativas de composición, para luego tomar decisiones, y parten del supuesto de un depósito de \$1’000,000.oo a un año.

SOLUCIÓN: Para resolver el problema, la ecuación a utilizar es: $\mathrm{F}=\mathrm{P}\left(\mathbf{1}+\frac{\boldsymbol{i n}}{\boldsymbol{m}}\right)^{\boldsymbol{m}}\{16\}$, que se puede encontrar en el libro de Bittinger, M. L.; [4]. Luego, se sugiere identificar cada variable conocida y la que se busca descubrir:

$$
\begin{aligned}
& \begin{array}{l}
\mathrm{P}=\$ 1^{\prime} 000,000 . \mathrm{oo} \\
\text { Tiempo }=\text { Un año }
\end{array} \quad \text { in }=0.15 \quad \mathrm{~m}=1 \quad \mathrm{~F}=? \\
& \mathrm{~F}=\$ 1^{\prime} 000,000 . \mathrm{oo}\left(\mathbf{1}+\frac{\mathbf{0 . 1 5}}{\mathbf{1}}\right)^{\mathbf{1}}=\$ 1^{\prime} 150,000.00
\end{aligned}
$$

Composición $=$ Semestral, Tiempo $=$ Un año in $=0.15$ $\mathrm{m}=2 \quad \mathrm{~F}=$ ?

$$
\mathrm{F}=\$ 1^{\prime} 000,000 . \mathrm{oo}\left(\mathbf{1}+\frac{\mathbf{0 . 1 5}}{\mathbf{2}}\right)^{2}=\$ 1^{\prime} 155,625 . \mathrm{oo} .
$$

$\mathrm{P}=\$ 1^{\prime} 000,000.00$

Composición $=$ Tetramestral

Tiempo $=$ Un año in $=0.15 \quad \mathrm{~m}=3 \quad \mathrm{~F}=$ ?

$$
\mathrm{F}=\$ 1^{\prime} 000,000 . \mathrm{oo}\left(\mathbf{1}+\frac{\mathbf{0 . 1 5}}{\mathbf{3}}\right)^{3}=\$ 1^{\prime} 157,625.00
$$

$$
\mathrm{P}=\$ 1^{\prime} 000,000.00 \quad \text { Composición }=\text { Trimestral }
$$$$
\text { Tiempo }=\text { Un año in }=0.15 \quad \mathrm{~m}=4 \quad \mathrm{~F}=\text { ? }
$$

$$
\mathrm{F}=\$ 1^{\prime} 000,000 . \mathrm{oo}\left(\mathbf{1}+\frac{\mathbf{0 . 1 5}}{\mathbf{4}}\right)^{\mathbf{4}}=\$ 1^{\prime} 158,650.42
$$

$\mathrm{P}=\$ 1^{\prime} 000,000 . \mathrm{oo} \quad$ Composición $=$ Bimestral

Tiempo $=$ Un año in $=0.15 \quad \mathrm{~m}=6 \quad \mathrm{~F}=?$

$$
F=\$ 1^{\prime} 000,000.00\left(\mathbf{1}+\frac{\mathbf{0 . 1 5}}{\mathbf{6}}\right)^{\mathbf{6}}=\$ 1^{\prime} 159,693.42 .
$$

$\mathrm{P}=\$ 1^{\prime} 000,000.00 \quad$ Composición $=$ Mensual

Tiempo $=$ Un año $\quad$ in $=0.15 \quad \mathrm{~m}=2 \quad \mathrm{~F}=$ ?

$$
\mathrm{F}=\$ 1^{\prime} 000,000.00\left(\mathbf{1}+\frac{\mathbf{0 . 1 5}}{\mathbf{1 2}}\right)^{\mathbf{1 2}}=\$ 1^{\prime} 160,754.52
$$

$\mathrm{P}=\$ 1^{\prime} 000,000.00 \quad$ Composición $=$ Semanal

Tiempo $=$ Un año in $=0.15 \quad \mathrm{~m}=52 \quad \mathrm{~F}=$ ?

$$
\mathrm{F}=\$ 1^{\prime} 000,000 . \mathrm{oo}\left(\mathbf{1}+\frac{\mathbf{0 . 1 5}}{\mathbf{5 2}}\right)^{\mathbf{5 2}}=\$ 1^{\prime} 161,583.39 .
$$

$\mathrm{P}=\$ 1^{\prime} 000,000.00 \quad$ Composición $=$ Diaria

Tiempo $=$ Un año in $=0.15 \quad \mathrm{~m}=365 \quad \mathrm{~F}=$ ?

$\mathrm{F}=\$ 1^{\prime} 000,000.00\left(\mathbf{1}+\frac{\mathbf{0 . 1 5}}{\mathbf{3 6 5}}\right)^{365}=\$ 1^{\prime} 161,798.44$.

$\mathrm{P}=\$ 1^{\prime} 000,000.00 \quad$ Composición $=$ Por horas

Tiempo $=$ Un año in $=0.15 \quad \mathrm{~m}=8760 \quad \mathrm{VF}=?$

$$
\mathrm{F}=\$ 1^{\prime} 000,000 . \mathrm{oo}\left(\mathbf{1}+\frac{\mathbf{0 . 1 5}}{\mathbf{8 7 6 0}}\right)^{\mathbf{8 7 6 0}}=\$ 1^{\prime} 161,831.86
$$

$\mathrm{P}=\$ 1^{`} 000,000.00 \quad$ Composición $=$ Continua

Tiempo $=$ Un año in $=0.15$

$\mathrm{m}=$ Indeterminada $\quad \mathrm{F}=$ ?

Aquí se utiliza la siguiente igualdad: $F=P\left(\mathbf{e}^{i n * t}\right)\{17\}$. Que se ha tomado del libro de Aguirre, V. H. M. [10].

$$
\mathrm{F}=\$ 1^{\prime} 000,000 . \mathrm{oo}\left(\mathbf{e}^{\mathbf{0 . 1 5}}\right)=\$ 1^{\prime} 161,834.24
$$

Al observar los resultados (F) en los diferentes ítems, se verifica la importancia o impacto de la capitalización debido a la composición. Los resultados anteriores se pueden resumir en el gráfico \#3. 
Tabla \#2 Periodos en un año, de acuerdo a la composición y el valor futuro equivalente, dado un valor presente de $\$ 1^{\prime} 000,000.00$

\begin{tabular}{|l|l|}
\hline $\mathrm{N}$ & $\mathrm{F}($ en miles $)$ \\
\hline 1 & $1^{\prime} 150,000 . \mathrm{oo}$ \\
\hline 2 & $1^{\prime} 155,625.00$ \\
\hline 3 & $1^{\prime} 157,625.00$ \\
\hline 4 & $1^{\prime} 158,650.42$ \\
\hline 6 & $1^{\prime} 159,693.42$ \\
\hline 12 & $1^{\prime} 160,754.52$ \\
\hline 52 & $1^{\prime} 161,583.39$ \\
\hline 365 & $1^{\prime} 161,798.44$ \\
\hline 8760 & $1^{\prime} 161,831.86$ \\
\hline
\end{tabular}

Gráfico 3: $\lim _{n \rightarrow \infty}\left(1+\frac{0.15}{n}\right)^{n}=\$ 1^{\prime} 161,831.86$

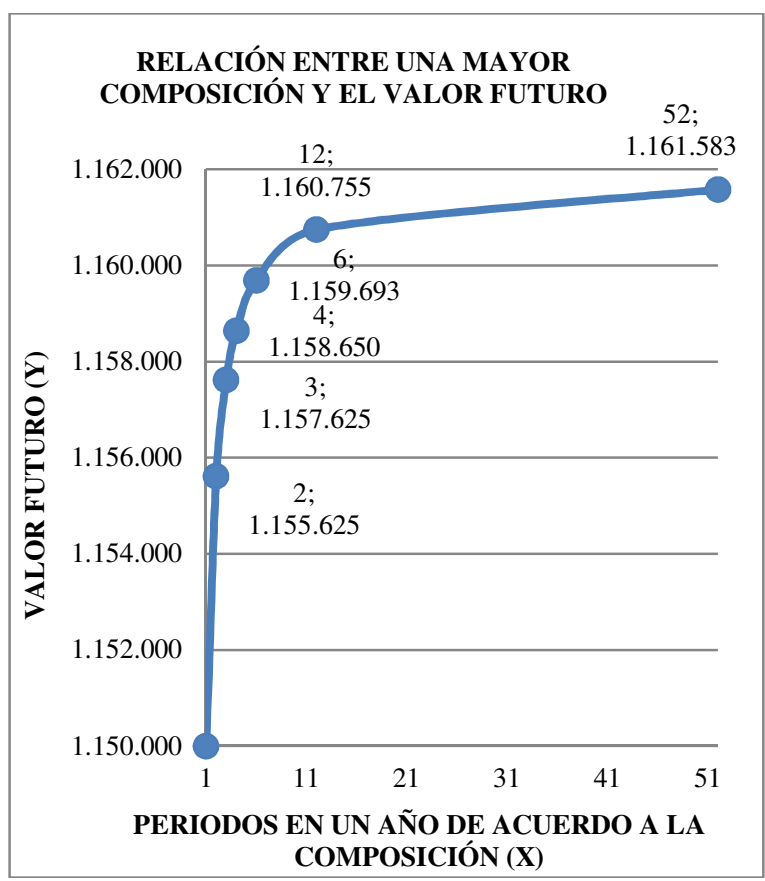

Ejemplo 2: Un inversionista cuenta con $\$ 5^{\prime} 000,000.00$ y ha recibo una propuesta de una banco de pagarle una tasa nominal del $14 \%$ de composición bimensual durante dos años; él desea saber en cuanto tiempo recibirá la misma cantidad como compensación en otra entidad financiera, que paga la misma tasa de interés pero continuo.

Solución: Se pasa a identificar variables conocidas y la que se debe esclarecer.

$\mathrm{P}=\$ 5^{\prime} 000,000.00 \quad \mathrm{~m}=6 \quad$ in $=14 \%$

Composición $=$ Bimensual $\quad$ Tiempo $=2$ años $. \quad F=?$
$\mathrm{F}=\$ 5^{\prime} 000,000.00\left(\mathbf{1}+\frac{\mathbf{0 . 1 4}}{\mathbf{6}}\right)^{(\mathbf{6} * \mathbf{2})}=\$ 6^{\prime} 594,402.53$

Ahora, se resolverá la otra parte, es decir, determinar el tiempo que tomará obtener el mismo $\mathrm{F}$, en interés continuo:

$$
\begin{gathered}
\$ 5^{\prime} 000,000.00\left(\mathbf{e}^{[\mathbf{0 . 1 4} * \boldsymbol{T}]}\right)=\$ 6^{\prime} 594,402.53 \\
\left(\mathbf{e}^{[\mathbf{0 . 1 4 * T}]}\right)=\frac{\$ \mathbf{6}^{\prime} \mathbf{5 9 4}, \mathbf{4 0 2 . 5 3}}{\mathbf{5}^{\prime} \mathbf{0 0 0}, \mathbf{0 0 0 . 0 0}} \\
\operatorname{Ln}\left(\mathbf{e}^{[\mathbf{0 . 1 4 * T}]}\right)=\ln 1.3188805 \rightarrow \\
0.14 * \mathrm{~T}=0.2767832 \quad \rightarrow \mathrm{T}=1.9770234
\end{gathered}
$$

El resultado indica que es un año más una parte porcentual del año, para determinar el tiempo en meses, se multiplica por los 12 meses que tiene el año.

$$
0.9770234 * 12=11.7242807
$$

Y la fracción posterior a 11, se multiplica por 30 para saber qué cantidad de días son equivalentes a ese decimal:

$$
0.7242807 * 30=21.7284206
$$

Las cifras permiten concluir que una tasa de interés del 14\% nominal durante dos años de composición bimensual tiene el mismo rendimiento que la mencionada tasa de composición continua aplicada durante un año, 11 meses y 22 días aproximadamente.

Ejemplo 3: Un empresario cuenta con $\$ 10^{\prime} 000,000$.oo y ha decidido depositarlos durante año y medio en una entidad financiera que paga una tasa nominal del $16 \%$ de composición mensual; el gerente de la competencia se ha enterado, y dado que tienen una promoción de interés continuo, va a ofrecerle una tasa inferior, pero que por efecto de la composición, sea más rentable, que la de la competencia. ¿Cuál es la tasa mínima que debe aceptar el empresario?

Solución:

Primera opción:

$\mathrm{P}=\$ 10^{\prime} 000,000.00 \quad$ in $=16 \% \quad \mathrm{~m}=12$

Composición $=$ Mensual $\quad$ Tiempo $=1.5$ años $\quad$ VF $=$ ?

Reemplazando en la ecuación:

$$
\mathrm{F}=\$ 10^{\prime} 000,000.00\left(\mathbf{1}+\frac{\mathbf{0 . 1 6}}{\mathbf{1 2}}\right)^{\{\mathbf{1 2 * 1 . 5}\}}=\$ 12^{\prime} 692,346.5
$$

Segunda opción:

$\mathrm{P}=\$ 10^{\prime} 000,000$.oo $\mathrm{m}=$ Indeterminada Composición $=$ Continua Tiempo $=1.5$ años 


$$
\begin{aligned}
& \mathrm{VF}=\$ 12^{\prime} 692,346.5 \quad \text { in }=? \\
& \$ 10^{\prime} 000,000.00\left(\mathbf{e}^{[i n * 1.5]}\right)=\$ 12^{\prime} 692,346.5 \\
& \left(\mathbf{e}^{[i n * 1.5]}\right)=\frac{\$ 12^{\prime} 692,346.5}{\$ 10^{\prime} 000,000.00} \\
& \operatorname{Ln}\left(\mathbf{e}^{[\boldsymbol{i n} * \mathbf{1 . 5}]}\right)=\ln 1.2692347 \\
& (\text { in } * 1.5)=\ln 1.2692347=0.2384141 \mathrm{i}
\end{aligned}
$$

in $=0.1589427$.

Dada la oferta de la segunda opción donde se compensan los ahorros con interés continuo, la tasa mínima que debería recibir el empresario, para no perder con respecto a la primera opción, es de $15.89 \%$

Ejemplo 4: La empresa HERMES, transportes y acarreos, desea contar de aquí a 21 meses con $\$ 75^{\prime} 254,361.65$ para comprar un nuevo vehículo (camión) de transporte de mercancía, la entidad cuenta en el momento con un excedente de tesorería, el cual calcula, que de ser colocado en un banco que paga interés nominal del $12 \%$ continuo, alcanzaría para a la fecha determinada, retirar el monto que se proyecta costará el camión. ¿De cuánto debe ser el depósito hoy?

Solución: En este caso, es necesario utilizar la siguiente fórmula: $\mathrm{P}=\mathrm{F}\left(\mathbf{e}^{-[i n * t]}\right) \quad\{18\}$, ilustrada por Varela V. R. [11].

Luego se pasa a detallar la información e identificar las variables: $\mathrm{F}=\$ 75^{\prime 2} 254,361.65$

Tiempo $=21$ meses (1.75 años)

Composición $=$ Continua in $=12 \%$ $\mathrm{m}=$ Indeterminada $\mathrm{P}=$ ?

$$
\mathrm{P}=\$ 75^{\prime} 254,361.65\left(\mathbf{e}^{-[\mathbf{0 . 1 2 * 1 . 7 5}]}\right)=\$ 61^{\prime} 000,000.00
$$

\section{CONCLUSIONES}

Al tratar los temas de cálculo financiero, regularmente se encuentra en los textos unas igualdades para aplicar, pero que el lector, de ser curioso o querer establecer el origen de las fórmulas, no puede profundizar sobre las mismas, ante esta circunstancia, se hace un humilde aporte tanto a las matemáticas como a las finanzas al argumentar, ilustrar y demostrar, como las dos áreas del conocimiento tienen una innegable afinidad por cuanto una se sirve de la otra, para manejar y resolver determinadas situaciones; así mismo es relevante que la comunidad encuentre un material que de forma práctica devele la pertinencia de utilizar ciertas ecuaciones en atención al tipo de operación financiera a valorar.

\section{REFERENCIAS}

[1] Sobel, M. A. y Lerner N. (1989). Algebra, (segunda edición, página 377). Editorial Prentice Hall.

[2] Thomas, Jr. G. B. (2005). Cálculo de una variable, (undécima edición, página 477). Pearson Educación.

[3] Hoffmann, L. D. (1990) Cálculo aplicado para administración, economía contaduría y ciencias sociales, (primera edición, página 171-174). Editorial McGraw-Hill.

[4] Bittinger, M. L. (2002). Cálculo para ciencias económico-administrativas, (séptima edición, página 292 a 293). Editorial Addison Wesley.

[5] Domínguez, Carlos. (2009). Manual de cálculo financiero, (primera edición, página 30). Editorial Villa María: Eduvim: Universidad Nacional de Rio Cuarto.

[6] Corredores y asociados S. A. (1994). Manual para el cálculo de rentabilidades, (cuarta edición, página 7). Serviflash Ltda.

[7] Vélez, P. I. (2006). Decisiones de inversión para la valoración financiera de proyectos y empresas, (quinta edición, página 81). Editorial Pontificia Universidad Javeriana.

[8] Aching, G. C. (2006). Matemáticas financieras para toma de decisiones empresariales. Recuperado el 20 de diciembre del 2011, de http://books.google.com.co/booksmatematicasfinancieras/mat ematicafinancieras (página 5).

[9] Haeussler E. F. Jr. y Richard, S. P. (2003). matemáticas para administración y economía, (decimoquinta edición, página 419). Pearson, Prentice Hall editores.

[10] Aguirre, V. H. M. (2008). Matemáticas financieras, (tercera edición, página 234). Cengage Learning editores.

[11] Varela, V. R. (1993). Evaluación económica de inversiones, (primera reimpresión, página 85). Grupo Editorial Norma. 